\title{
Parameter Study of Hemodynamics Simulation at Internal Carotid Stenosis*
}

\author{
Toshio NAKAYAMA**, Hitoshi HAYASE***, Koji TOKUNAGA*** \\ and Makoto OHTA**** \\ **Institute of Fluid Science, Tohoku University \\ 2-1-1 Katahira, Aoba-ku, Sendai, Miyagi 980-8577, Japan. \\ E-mail:nakayama@biofluid.ifs.tohoku.ac.jp \\ *** Neurological Surgery, Okayama University Graduate School of Medicine, \\ Dentistry and Pharmaceutical Sciences \\ 2-5-1 Shikata-cho, Okayama, Okayama 700-8558, Japan \\ **** Institute of Fluid Science, Tohoku University \\ 2-1-1 Katahira, Aoba-ku, Sendai, Miyagi 980-8577, Japan
}

\begin{abstract}
Background and purpose: Stenting is one of the treatment options for carotid artery stenosis. To show the effectiveness of the treatment, computational fluid dynamics (CFD) has been performed for the last several decades. The inlet/outlet boundary conditions are important in CFD, and several researchers have used various inlet/outlet boundary conditions. In this paper, we compared the blood flow with various inlet/outlet boundary conditions and the measurement data obtained by an ultrasound device.

Methods: Blood flow speed was measured in the internal carotid artery (ICA), the external carotid artery (ECA), and the common carotid artery (CCA) using an ultrasound device. A carotid artery was reconstructed by computed tomography (CT). Mesh in the integrated data was generated to perform flow dynamics using a commercial code. CFD for blood flow was performed using the reconstructed carotid artery. Five cases of inlet and outlet boundary conditions (I/O B.C.) were used for the CFD. The simulation results were compared with the ultrasound data on the blood flow speed in the vicinity of the center of the ICA and the ECA.

Results: Various blood flow speeds were obtained from the five cases. The case of adjustment of pressures in ICA and ECA is the nearest flow speed to the ultrasound data.

Conclusion: The flow speed depends on the I/O B.C.. The I/O B.C. may be necessary for the measurement data obtained by ultrasound device or magnetic resonance imaging (MRI).
\end{abstract}

Key words: Stent, Hemodynamic, Carotid Artery, Stenosis, FVM, CFD

\section{Introduction}

The Cholesterols and the thrombosis pile up easily in the internal carotid artery (ICA) ${ }^{(1)}$. The ICA is one of the areas of predilection for stenosis ${ }^{(1)}$. Such stenosis may cause the decrease in cerebral blood flow and the infarct cerebral ${ }^{(1)}$. Poepping et al. ${ }^{(2)}$ measured the blood flow in the carotid artery using an ultrasound device and described an in vitro system developed to obtain time-varying 3-D Doppler ultrasonic data in carotid artery bifurcation phantoms. Tada et al. ${ }^{(3)}$ presented a three-dimensional steady numerical simulation of flow using a model of the bifurcation of the common carotid artery (CCA) into the ICA and

*Received 24 Jan., 2008 (No. 08-0044) [DOI: 10.1299/jfst.3.544] 
external carotid artery (ECA). Flow with large inertia bifurcates at the apex and creates a flow recirculation zone associating with very low wall shear stress at the outer wall of the ICA sinus where atherosclerotic plaque is localized. The change of both the CCA-ICA and CCA-ECA bifurcation angle greatly affects the distribution of Wall Shear Stress (WSS) on the outer wall of the sinus. Noda et al. (3) simulated the blood flow for carotid artery stenosis, and described a method of behavior forecast and collection debris.

The inlet and outlet boundary conditions (I/O B.C.) in a bifurcated pipe may be an important issue for producing an effective flow bifurcation ${ }^{(4)}$. On the other hand, as the blood volume flow rate has been already bifurcated in each patient, the boundary conditions are important for reproducing the blood flow pattern. Several previous researches (3), (5)-(7) have used the outlet pressure conditions. However, because it is too difficult to measure the blood pressure of the carotid artery, it was set at $0[\mathrm{~Pa}]$ in several researches. Using this condition, the difference between the measurements and the simulation results will occur. To solve this problem, the velocity outlet condition was used. Tada et al. ${ }^{(2)}$ used the flow rate for the outlet boundary condition. Birchall et al. ${ }^{(8)}$ used the flow rate for the boundary conditions of the CCA and ICA. They have used flow rate that were calculated from measurements. Tori et al. ${ }^{(9)}$ used the velocity inlet condition and outlet condition that considers the influence of the distal arterial network.

Although many researchers used various boundary conditions, the qualities of these conditions have not been discussed. In the present research, first, blood velocities were measured at three places (the ICA, the ECA, and the CCA) using an ultrasound measurement device. By comparing the ultrasound data with the simulation results, we look for the most suitable boundary condition for carotid artery simulation.

\section{Materials and Methods}

3-D Reconstruction of a carotid artery

Three-dimensional reconstruction of a carotid artery of a patient (male, 65-year-old) was performed from medical images using a computed tomography (CT) system. The parameters of the CT image are show in Table 1.

Table 1 CT Image Parameters

\begin{tabular}{|l|l|}
\hline Rows x Columns & $512 \times 512$ \\
\hline Pixel Spacing & $0.116[\mathrm{~mm}]$ \\
\hline Slice Thickness & $1[\mathrm{~mm}]$ \\
\hline No. Images & 106 \\
\hline Direction & axial \\
\hline
\end{tabular}

The three-dimensional shape was generated by accumulating the CT slice images. We extracted the region of blood from the DICOM image. The carotid artery shape was edited and reconstructed using Mimics (7.30, Materialise, Belgium). As the data output from mimics had a rough surface and poorly shaped triangles, the surface shape data were adjusted by smoothing and a triangle reduction using Magics (8.05, Materialise, Belgium). When the shape was restructured, the volume was decreased by $0.5 \%$ or less. The output data were in the STL format.

The reconstructed shape data were transferred to a supercomputer (Silicone Graphics Prism, SGI Japan, Ltd, Japan) and a tetrahedron numerical mesh was generated using a commercial software package (Gambit 2.2.30, Fluent Inc., NH). 


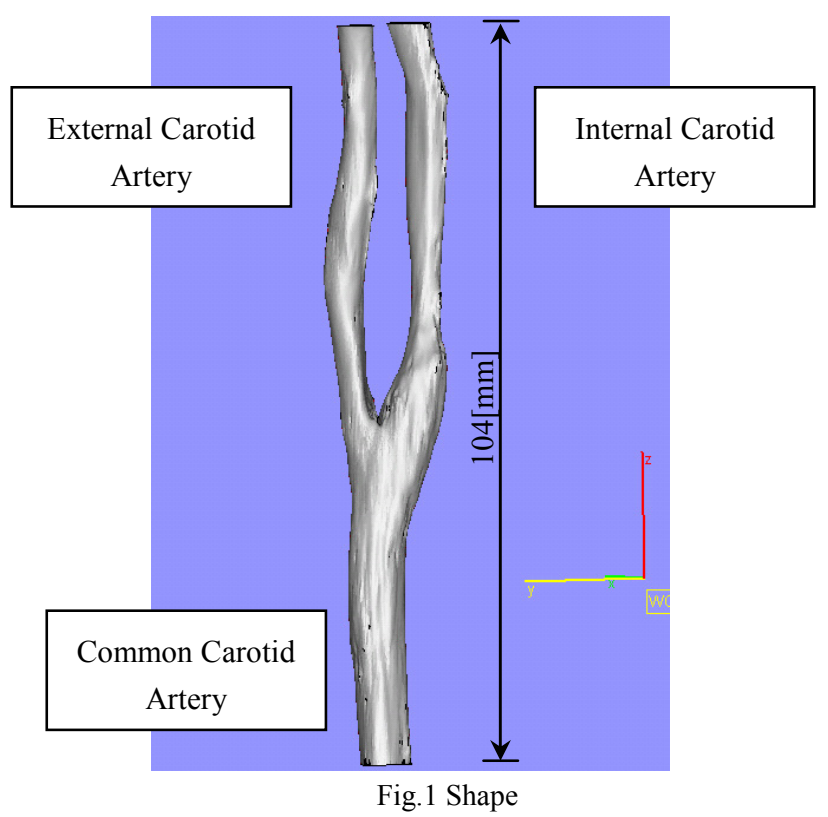

\section{Numerical Simulation}

The flow speed proportion (the blood flow speed ratio of ICA and ECA) was introduced, and then the blood flow in the carotid artery was evaluated using the flow speed proportion of the ultrasound measurement and simulation results. The flow speed proportion was defined as follows.

$$
\text { Flow speed proportion }=\frac{\text { The blood flow speed at center of internalcarotid artery }}{\text { The blood flow speed at center of externalcarotid artery }}
$$

The ultrasound procedure was carried out as routine clinical diagnostic practice. The measurement point of blood flow speed was set as follows. The point in the ICA was 0.02 $[\mathrm{m}]$ after the bifurcation. The point in the ECA was the narrowest part after the bifurcation. The point of the CCA was $0.02[\mathrm{~m}]$ before the bifurcation. The ultrasound data were 1.124 $[\mathrm{m} / \mathrm{s}]$ at the ICA measurement point, and $1.094[\mathrm{~m} / \mathrm{s}]$ at the ECA measurement point. Thus, the flow speed proportion was 1.027 .

The blood flow speed at the I/O B.C. was calculated based on the ultrasound data. At first, the ultrasound data were assumed to be the maximum blood flow speed at the measurement points, and calculated flow rate. The mean blood flow speed was then calculated using those flow rates. A blood flow speed of $0.677[\mathrm{~m} / \mathrm{s}]$ was set in inlet, a blood flow speed of $0.407[\mathrm{~m} / \mathrm{s}]$ was set in outlet of ICA, and a blood flow speed of 0.607 $[\mathrm{m} / \mathrm{s}]$ was set in outlet of ECA.

Table 2 Boundary Conditions

\begin{tabular}{|l|c|c|c|c|c|}
\hline & Case 1 & Case 2 & Case 3 & Case 4 & Case 5 \\
\hline Inlet & velocity & velocity & pressure & velocity & velocity \\
(CCA) & $0.677[\mathrm{~m} / \mathrm{s}]$ & $0.677[\mathrm{~m} / \mathrm{s}]$ & $0[\mathrm{~Pa}]$ & $0.677[\mathrm{~m} / \mathrm{s}]$ & $0.677[\mathrm{~m} / \mathrm{s}]$ \\
\hline Outlet & pressure & pressure & velocity & pressure & velocity \\
(ICA) & $0[\mathrm{~Pa}]$ & $178.01[\mathrm{~Pa}]$ & $0.407[\mathrm{~m} / \mathrm{s}]$ & $0[\mathrm{~Pa}]$ & $0.407[\mathrm{~m} / \mathrm{s}]$ \\
\hline Outlet & pressure & pressure & velocity & velocity & pressure \\
(ECA) & $0[\mathrm{~Pa}]$ & $0[\mathrm{~Pa}]$ & $0.607[\mathrm{~m} / \mathrm{s}]$ & $0.607[\mathrm{~m} / \mathrm{s}]$ & $0[\mathrm{~Pa}]$ \\
\hline
\end{tabular}


We examined the relation between the blood flow speed in the carotid artery and the I/O B.C.. Three categories, namely, pressure outlets, velocity outlets, and mixture of pressure and velocity outlets, were separated into five cases as follows.

(1) Pressure outlet category

Case 1: Pressure of $0[\mathrm{~Pa}]$ was set at the ICA and ECA outlets. Blood flow speed of $0.677[\mathrm{~m} / \mathrm{s}]$ was set at the inlet.

Case 2: Pressure of $178.01[\mathrm{~Pa}]$ and $0[\mathrm{~Pa}]$ were set at the ICA outlet and the ECA outlet, respectively. Blood flow speed of $0.677[\mathrm{~m} / \mathrm{s}]$ was set at the inlet.

The pressure of $178.01[\mathrm{~Pa}]$ was determined by the flow speed proportion described by Eq. (1) and Fig. 2. To obtain the same proportion as that of ultrasound data, pressure of 70, $140,210[\mathrm{~Pa}]$ were first set at the ICA outlet, respectively, and the pressure at the ECA outlet was always $0[\mathrm{~Pa}]$. The flow speed proportion was then calculated so as to be the same as that of ultrasound data.

Figure 2 is a graph of the ICA outlet pressure and flow speed proportion. The intersection of the blue line and the red line may be the appropriate pressure of the ICA. From this figure, the pressure of ICA was $178.01[\mathrm{~Pa}]$.

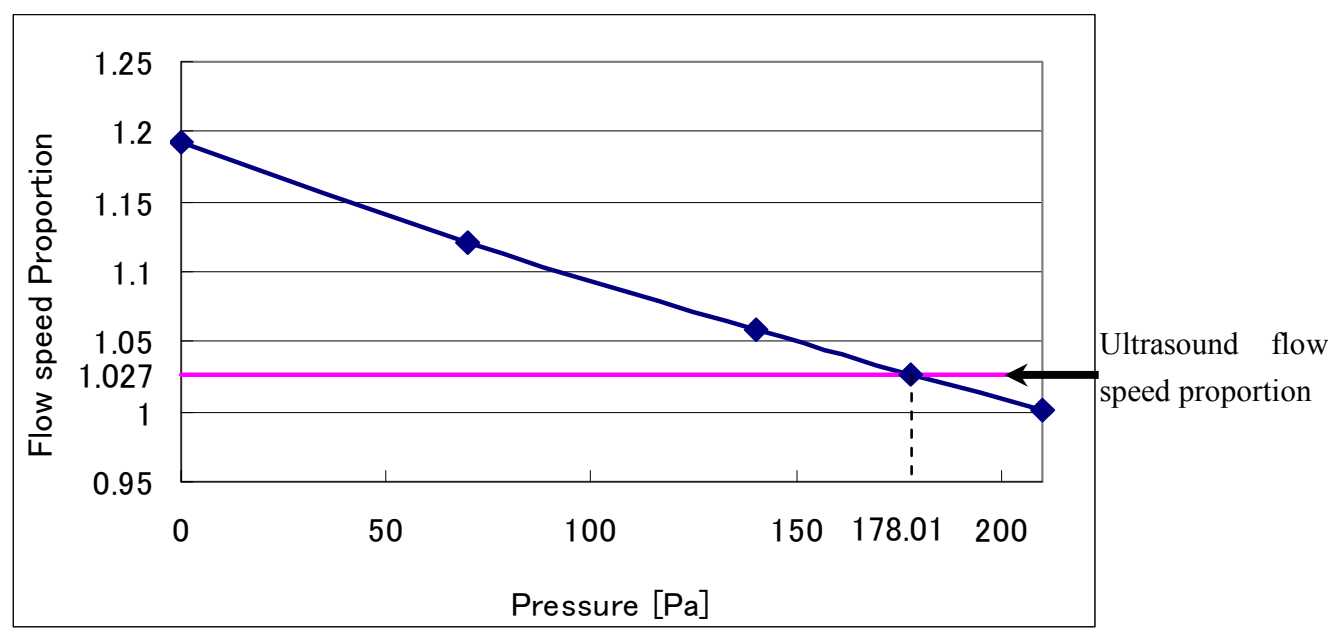

Fig. 2 Flow speed proportion (CFD and Ultrasound)

(2) Velocity outlet category

Case 3: A blood flow speed of 0.407 [m/s] was set at outlet of the ICA and a blood flow speed of $0.607[\mathrm{~m} / \mathrm{s}]$ was set at the outlet of the ECA. A pressure of $0[\mathrm{~Pa}]$ was set at the inlet.

(3) Pressure outlet \& velocity outlet category

In these cases, either the outlet boundary condition of the ICA or the ECA was set as the velocity condition, the other outlet condition was set as the pressure condition.

Case 4: Pressure of $0[\mathrm{~Pa}]$ was set at the ICA outlet and blood flow speed of $0.607[\mathrm{~m} / \mathrm{s}]$ was set at the ECA outlet. Blood flow speed of $0.677[\mathrm{~m} / \mathrm{s}]$ was set at the inlet.

Case 5: Blood flow speed of $0.407[\mathrm{~m} / \mathrm{s}]$ was set at the ICA outlet and pressure of 0 $[\mathrm{Pa}]$ was set at the ECA outlet. Blood flow speed of $0.677[\mathrm{~m} / \mathrm{s}]$ was set at the inlet.

We simulated five cases with the above-mentioned parameters.

The blood flow was simplified as isothermal, incompressible and laminar Newtonian flow with a density of $1050\left[\mathrm{~kg} / \mathrm{m}^{3}\right]$ and a viscosity of $0.0035[\mathrm{~Pa} \mathrm{~s}]$.

As the steady simulation was performed, the boundary conditions of the inlet, outlet, and vessel wall were time-independent. As the target of the simulation was the blood flow analysis at the systolic duration, a flat flow velocity profile with $0.677[\mathrm{~m} / \mathrm{s}]$ was introduced on the inlet to be consistent with the Reynolds Number of 1730. The wall boundary 
condition of the blood vessel wall was set No-slip condition.

The basic equations of continuity and the Navier-Stokes equation were solved to determine the flow field. The calculation using the finite volume method was performed by the commercial solver of Fluent 6.2.16 (Fluent Inc., NH) installed in Prism (SGI Japan, Ltd, Japan). The number of total meshes became about 1,300,000 elements.

\section{Results}

Case 1: The I/O B.C. were set by pressure outlet and velocity inlet. Figure 3 shows the velocity contour at the ICA and ECA measurement points. Figure 4 shows the streamline. The colors indicate the speed of blood flow, blue being slow speed and red being fast speed. The speed of blood flow in the center of the ICA and in the center of the ECA were 0.933 $[\mathrm{m} / \mathrm{s}]$ and $0.783[\mathrm{~m} / \mathrm{s}]$, respectively. The blood speed of the ICA is faster than that of the ECA. The relation between the speed of blood flow of the ICA and that of the ECA is similar to that of the ultrasound data. The flow speed proportion was 1.19. The difference of blood flow speed between the simulation results and the measurement data in the ICA and ECA are $17.00 \%$ and $28.44 \%$, respectively.

Case 2: Pressure of $178.01[\mathrm{~Pa}]$ and $0[\mathrm{~Pa}]$ were set at the ICA outlet and ECA outlet, respectively. Velocity of 0.677 [m/s] was set at inlet. Figure 5 shows the velocity contour at the ICA and ECA measurement points. Figure 6 shows the streamline. The colors indicate the blood flow speed. This case used the same flow speed proportion. The blood flow speed in the center of the ICA and in the center of the ECA were $1.08[\mathrm{~m} / \mathrm{s}]$ and $1.06[\mathrm{~m} / \mathrm{s}]$, respectively. The flow speed proportion was 1.025 . The difference of blood flow speed between the simulation results and the measurement data in the ICA and ECA were 3.67\% and $3.48 \%$, respectively.

Case 3: The blood flow speeds were set at the outlets boundary and pressure was set inlet. Figure 7 shows the velocity contour at the ICA and ECA measurement points. Figure 8 shows the streamline. The colors indicate the blood flow speed. The blood flow speed of the ICA is slower than that of the ECA. The relation between the blood flow speed in the ICA and that in the ECA is opposite to that of the ultrasound data. The blood flow speed in the center of the ICA and ECA were $0.825[\mathrm{~m} / \mathrm{s}]$ and $0.854[\mathrm{~m} / \mathrm{s}]$, respectively. The flow speed proportion was 0.955 . The difference of blood flow speed between simulation results and measurement data in the ICA and ECA are $26.65 \%$ and $17.02 \%$, respectively.

Case 4: The blood flow speed was set at the outlet of the ICA and pressure was set at the outlet of the ECA. Figure 9 shows the velocity contour at ICA and ECA measurement points. Figure 10 shows the streamline. The colors indicate the blood flow speed. The blood flow speed in the center of the ICA and the ECA are $0.800[\mathrm{~m} / \mathrm{s}]$ and $1.29[\mathrm{~m} / \mathrm{s}]$, respectively. The blood speed of ICA is slower than the blood flow speed of ECA. The relation between the blood flow speed in the ICA and that in the ECA is opposite to that of the ultrasound data. The flow speed proportion is 0.618 . The difference of blood flow speed between simulation results and measurement data in the ICA and ECA are $28.83 \%$ and $26.85 \%$, respectively.

Case 5: Pressure was set at the outlet of the ICA and the blood flow speed was set at the outlet of the ECA. The blood flow speed was set at inlet. Figure 11 shows the velocity contour at ICA and ECA measurement points. Figure 12 shows streamline. The colors indicate blood flow speed. The blood flow speed in the center of the ICA and the ECA were $1.31[\mathrm{~m} / \mathrm{s}]$ and $0.744[\mathrm{~m} / \mathrm{s}]$, respectively. The blood speed of ICA is faster than the blood flow speed of ECA. The relation between the ICA blood flow speed and the ECA blood flow speed is similar to that of the ultrasound data. The flow speed proportion is 1.76 . The difference of blood flow speed between the simulation results and measurement data in the ICA and ECA are $16.28 \%$ and $31.98 \%$, respectively. 


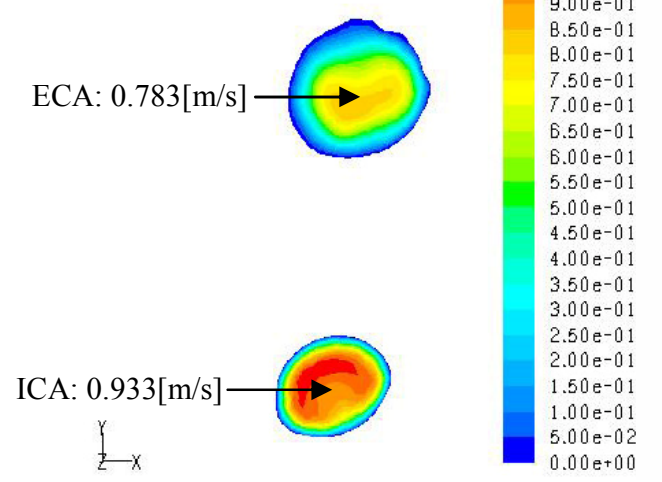

Fig.3 Velocity Contour

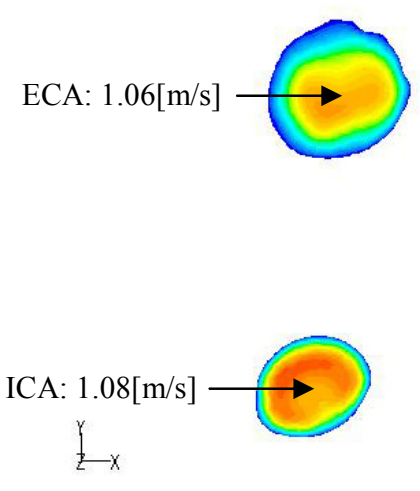

Fig. 5 Velocity Contour

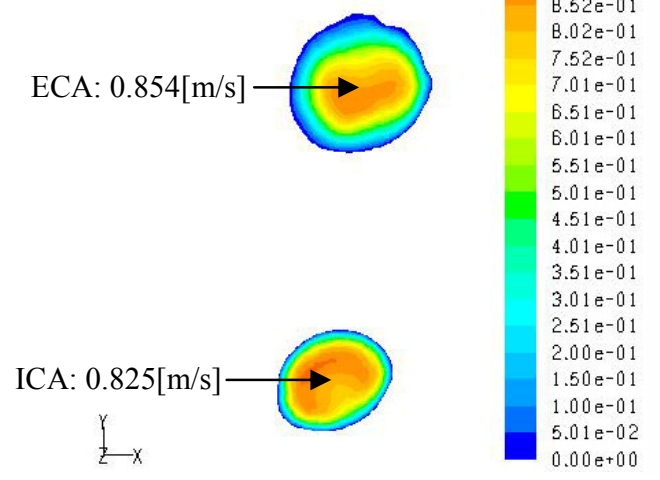

Fig.7 Velocity Contour

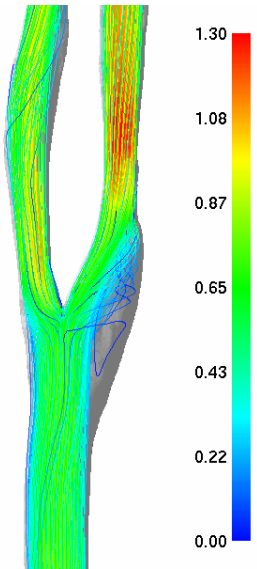

Fig.4 Streamline

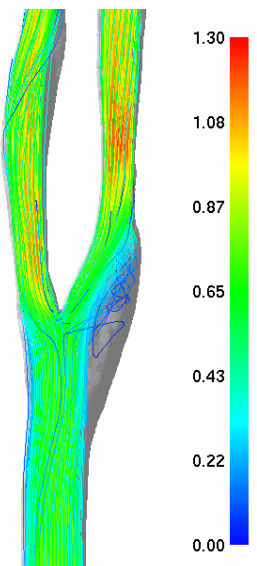

Fig.6 Streamline

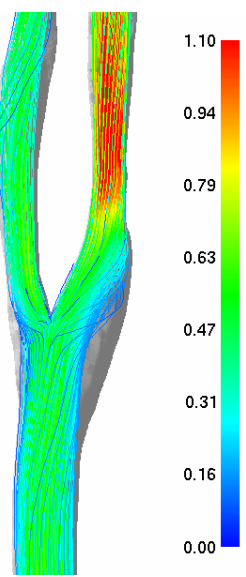

Fig.8 Streamline 


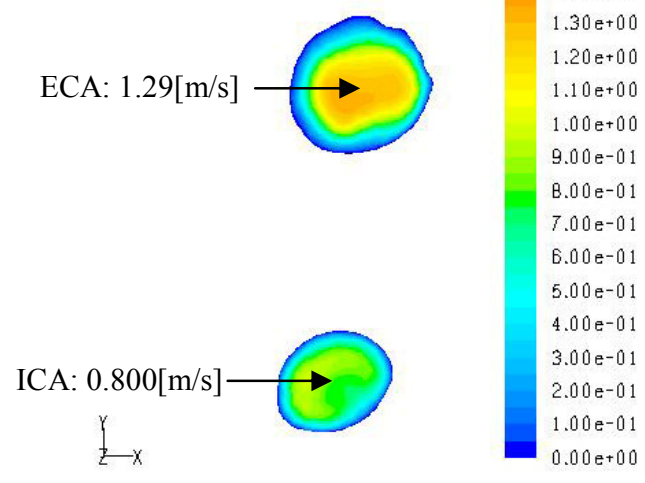

Fig.9 Velocity Contour

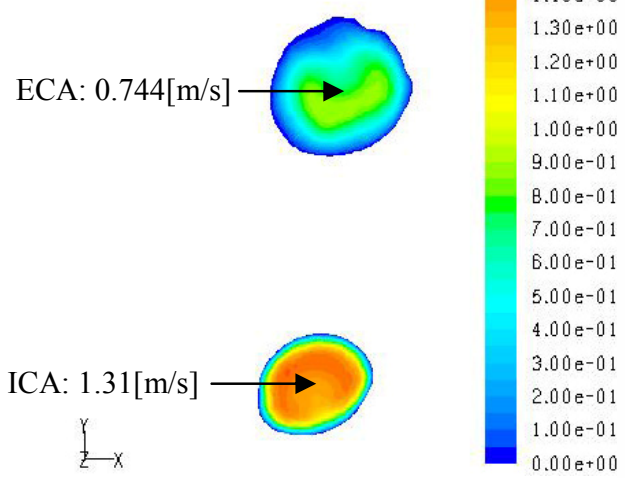

Fig.11 Velocity Contour

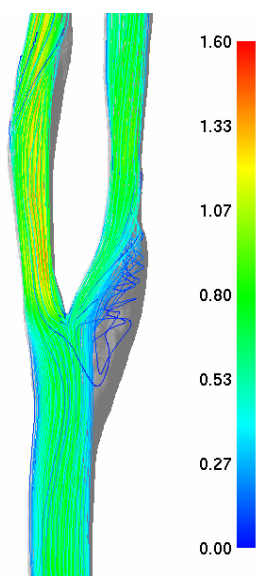

Fig.10 Streamline

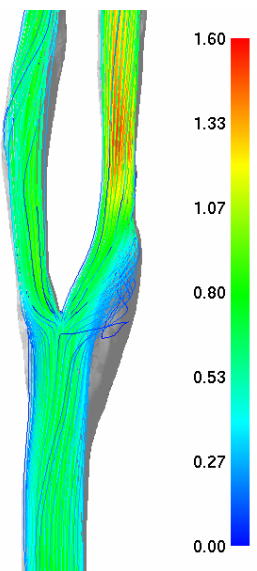

Fig.12 Streamline

Table 3 shows the blood flow speed of the ICA and the ECA and the flow speed proportions of all each cases. Table 4 shows the difference between the simulation results and the measurements data.

Table 3 Flow speed proportions

\begin{tabular}{|c|c|c|c|c|c|}
\hline. & Case 1 & Case 2 & Case 3 & Case 4 & Case 5 \\
\hline Velocity (ICA) $[\mathrm{m} / \mathrm{s}]$ & 0.933 & 1.08 & 0.825 & 0.800 & 1.31 \\
\hline Velocity (ECA) [m/s] & 0.783 & 1.06 & 0.854 & 1.29 & 0.744 \\
\hline Flow speed proportion & 1.19 & 1.03 & 0.955 & 0.618 & 1.76 \\
\hline
\end{tabular}

Table 4 The difference between simulation results and measurements data

\begin{tabular}{|c|c|c|c|c|c|}
\hline & Case 1 & Case 2 & Case 3 & Case 4 & Case 5 \\
\hline ICA [\%] & 17.00 & 3.67 & 26.65 & 28.83 & 16.28 \\
\hline ECA [\%] & 28.44 & 3.48 & 17.02 & 26.85 & 31.98 \\
\hline
\end{tabular}

The difference between the value of simulation results and those of the ultrasound measurement of ultrasound is under $4 \%$. The difference of the maximum velocity in the 
ECA can be decreased from $20 \%$ to $4 \%$ by adjusting the pressure at the outlet.

\section{Discussion}

\subsection{Accuracy of calculation}

In this simulation, the shapes of the simulated objects were complex. The blood vessel with the bifurcation was represented by realistic shapes at millimeter scale. Therefore, a tetrahedron mesh with a high space filling rate was selected. For a low Reynolds number such as that for the blood flow in a carotid artery, the calculation accuracy of a tetrahedron mesh was lower than that of a hexahedron mesh, and improvement of calculation accuracy will be necessary in the future.

Simulation was performed for all cases. The number of mesh elements of these cases was about 1.3 million. Although the arrangement of the tetrahedron mesh in these samples was not the same, the influence on simulation results by the difference of mesh number and arrangement of the tetrahedron mesh is thought to be small.

\subsection{Influence on calculation results by the difference of I/O B.C.}

In this study, we changed the I/O B.C. and investigated simulation results by difference of I/O B.C..

The outlet boundary conditions were at the set pressure outlet, the blood flow speed in the ICA was faster than that in the ECA. The flow speed balance of the ICA and ECA was equal to that of the ultrasound measurement results. The outlet boundary conditions were set the pressure and velocity outlet, the velocity of the pressure condition side was faster than the velocity condition side. The flow on the velocity condition side was not easy than the other side. The blood flow speed in the ICA and that in the ECA were greatly different from ultrasound measurements. The outlet boundary conditions were set at the velocity outlet, the flow speed balance of the ICA and the ECA was reversed. This was different from the ultrasound measurements. The ICA and ECA blood flow speed amount was slower than ultrasound measurement. From the view point of the blood flow speed and the flow speed balance of ICA and ECA, the best I/O B.C. may be Case 2 where the velocity inlet and optimal pressure outlet were set.

From the figure showing streamlines, the streamline in the carotid sinus is seen to be different due to the I/O B.C.. Rotational flow as secondary flow was occurred in the carotid sinus. We found that the size of rotational flow differed depending on the boundary conditions. Excluding Case 3, though the boundary condition of the inlet was the same, the size of the rotational flow was different. Even a main blood flow pattern of carotid artery, there was a difference in the blood flow pattern. We think that the careful boundary setting was necessary on the complex blood vessel shape.

The velocity boundary condition was calculated from the blood flow speed at the center of the blood vessel. The blood flow speed distribution in the blood vessel cross section was not obtained. The blood flow speed distribution may be necessary for the setting of velocity boundary conditions. If the blood flow speed distribution will be obtained using magnetic resonance angiography (MRA) device, we will be able to advance this research.

\section{Conclusion}

We simulated the blood flow in the carotid artery using several I/O B.C.. Simulation results were compared with ultrasound data and simulation results were different in all cases.

The setting of I/O B.C. made a great difference of the ultrasound measurement. The I/O B.C. may be necessary for the measurement data such as ultrasound device or MRI. 


\section{References}

(1) Noda S, Himeno R, Sasahara W, Behavior of the debris in the artery stenosis, Proceedings of the 19th Symposium on Computatuional Fluid Dynamics, CD-ROM, 2005.

(2) Poepping TL, Nikolov HN, Rankin RN, Lee M, Holdsworth DW, An in vitro system for Doppler ultrasound flow studies in the stenosed carotid artery bifurcation., Ultrasound Med Biol., Vol28, No.4, pp 495-506, 2002.

(3) Tada S, Tarbell JM, Computational Study of Flow in a Carotid Bifurcation: Geometry and Flow Ratio Correlations with WSS(Fluids Engineering), Transactions of the Japan Society of Mechanical Engineers. B, Vol.73, No.728, pp. 930-935, 2007. [Japanese]

(4) Naito T, Hayase T, Shirai A, Yamaguchi R, Numerical Simulation of Flow in Right Angle Branch : Mechanism of Periodical Flow Oscillation in Side Branch, JSME annual meeting, Vol.2000, No.1(20000731) pp. 257-258. [Japanese]

(5) Stuhne GR, Steinman1 DA, Finite-Element Modeling of the Hemodynamics of Stented Aneurysms, Journal of Biomechanical Engineering, Vol.126, pp381-390, 2004.

(6) Ohta M, Wetzel SG, Dantan P, Bachelet C, Lovblad KO, Yilmaz H, Flaud P, Rüfenacht DA, Rheological Changes After Stenting of a Cerebral Aneurysm: A Finite Element Modeling Approach, CardioVascular and Interventional Radiology, Vol. 28, pp. 768-772, 2005.

(7) Qiao A, Liu Y, Chang Y, Matsuzawa T. Computational Study of Stented Aortic Arch Aneurysms, Proceedings of the 2005 IEEE Engineering in Medicine and Biology 27th Annual Conference, pp.2287-2290, 2005.

(8) Birchall D, Zaman A, Hacker J, Davies G, Mendelow D, Analysis of haemodynamic disturbance in the atherosclerotic carotid artery using computational fluid dynamics, European Radiology, Vol.16, pp.1074-1083, 2006.

(9) Tori R, Oshima M, Kobayashi T, Takagi K, Tezduyar TE, Influence of wall elasticity in patient-specific hemodynamic simulations, Computers \& Fluids, Vol.36, Issue 1, pp. 160-168, 2007. 